\title{
A PDA-based system for online recording and analysis of concurrent events in complex behavioral processes
}

\author{
JÜRGEN HELD and TANJA MANSER \\ Swiss Federal Institute of Technology (ETH), Zurich, Switzerland
}

\begin{abstract}
This article outlines how a Palm- or Newton-based PDA (personal digital assistant) system for online event recording was used to record and analyze concurrent events. We describe the features of this PDA-based system, called the FIT-System (flexible interface technique), and its application to the analysis of concurrent events in complex behavioral processes-in this case, anesthesia work processes. The patented FIT-System has a unique user interface design allowing the user to design an interface template with a pencil and paper or using a transparency film. The template usually consists of a drawing or sketch that includes icons or symbols that depict the observer's representation of the situation to be observed. In this study, the FIT-System allowed us to create a design for fast, intuitive online recording of concurrent events using a set of 41 observation codes. An analysis of concurrent events leads to a description of action density, and our results revealed a characteristic distribution of action density during the administration of anesthesia in the operating room. This distribution indicated the central role of the overlapping operations in the action sequences of medical professionals as they deal with the varying requirements of this complex task. We believe that the FIT-System for online recording of concurrent events in complex behavioral processes has the potential to be useful across a broad spectrum of research areas.
\end{abstract}

Our objective is to demonstrate a mobile event recording system and its application to the online recording and analysis of concurrent events in complex behavioral processes. This recording system has been used successfully by researchers from a variety of backgrounds (e.g., engineering, psychology, and biology) for the online recording of event sequences in a variety of research contexts ranging from animal behavior to workplace design. To highlight the system's potential advantages when recording and analyzing concurrent events in complex behavioral processes, we use the example of work processes in the inherently complex work environment of surgical anesthesia.

Human activity in this context is much more complex than a sequence of single operations. In complex environments, several processes related to one or more goals take place in parallel. Even when focusing on processes that are directly observable, several forms of co-occurrence can be witnessed on the organizational and team levels,

The FIT-System PDA software is commercially available for Palm OS (trademark of PalmSource, Inc.) versions 3.0 and higher. Data transfer requires FIT-System PC software that is available for Windows OS (trademark of Microsoft, Inc.) versions 95 and higher. The authors retain a proprietary interest in the software described herein. T.M. is affiliated with the Institute of Work Psychology, J.H. with the Institute of Hygiene and Applied Physiology, both at ETH. Correspondence relating to this article may be sent to J. Held, Swiss Federal Institute of Technology, Clausiusstrasse 25, CH-8092 Zurich, Switzerland (e-mail: jheld@.ethz.ch).

Note-This article was accepted by the previous editor, Jonathan Vaughan. as well as on the level of individual task execution. Systematic investigation of the temporal structure of complex processes, therefore, should comprise the analysis of concurrent events.

Previous studies of anesthesia work processes have all faced the methodological problem of recording concurrent events online. In our research, however, we countered this problem by applying an observation method that is sensitive to concurrent events. This field study, conducted on the work processes relating to the administration of anesthesia (Manser, 2002, 2003; Manser \& Wehner, 2002), serves as an example to illustrate the use of the FIT-System with its specific graphical user interface and its syntax for event recording (Held, 1999) and data analysis.

\section{Concurrent Events in Anesthesiology Action Sequences: A Methodological Problem for Task Analysis}

Since the early 1970s, several systematic studies have been conducted on the individual task execution of anesthesiologists in operating theatres (e.g., Held, Brüesch, Zollinger, Pasch, \& Krueger, 2002; Kennedy, Feingold, Wiener, \& Hosek, 1976; Weinger, Herndon, \& Gaba, 1997; Weinger et al., 1994). These studies focused primarily on ergonomic concerns and were based on observations made either within the work setting or from filmed anesthetic procedures.

Because of the inconsistent assignment of observation categories among the various researchers, it seems almost 
impossible to compare the results of these different studies. Nevertheless, the debate on content, number, and resolution of observation codes has led to continuous improvements in the systematic structure and completeness of these codes. However, it has become obvious from these studies that methodological approaches based on a single-channel model of human activity (i.e., based on an assumption that one operation ends before another one starts) might oversimplify the structure of the complex action sequences of anesthesiologists. Thus, the ability to record and analyze concurrent events in action sequences-a core characteristic of practice when administering anesthesia-has been a major, recurring methodological problem.

One recording technique for concurrent events has been to toggle "between the two task categories based on the frequency each dominated the subject's time" (Weinger et al., 1994, p. 79). This solution, however, has led to new disadvantages concerning the analysis and interpretation of the data-for example, "the occurrence of simultaneous tasks ... will result in . . . decreased dwell time values (task duration)" (Weinger et al., 1994, p. 89).

These methodological problems demonstrate challenges in the development of an adequate methodological approach to human behavior in complex activity settings. In the following section, we describe a new method that is sensitive to concurrent events and that therefore provides a means to investigate this key aspect of complex behavioral processes.

\section{METHOD}

The following section describes the FIT-System for online event recording and illustrates its functionalities by presenting the example of a study of concurrent events in anesthesiology action sequences.

\section{The FIT System}

The FIT-System (flexible interface technique) is a patented computer-based method for the mobile recording of observable data
(Held, 1999, 2003). The hardware components are a hand-held computer device (a personal digital assistant, or PDA, either a device using the PalmOne OS or an Apple Newton Message Pad) and a paper or transparency template to use as an overlay covering the PDA's touch screen.

A main characteristic of the FIT-System is that its users can design their own interface on the transparency overlay or template. Thus, FIT-System users can decide which symbols and arrangements to use in their interaction with the event recorder (see Figures 1 and 2 for examples).

During an observation session, the FIT-System software incorporated into a PDA provides the user with the ability to record the start time of an identified event by tapping the related symbol with a stylus. The FIT-System provides auditory feedback (with adjustable loudness) for each data entry tap. In addition, the system allows the user to link additional information, such as handwritten notes, to the observation data by using the current count of an entered piece of data that is displayed on the PDA screen. The software stores the Cartesian coordinates of input data and the corresponding time codes to the PDA's memory. Therefore, task symbols on the event recorder interface do not have to be defined for the PDA's software, which makes the recording system more flexiblethis feature allows the user to adapt or enlarge the interface's set of symbols (e.g., draw new buttons) during the development and prototyping phases, and even in real time during online event recording. However, during actual study new observation codes must not be introduced, as this would affect the reliability of the data.

For data analysis, the FIT-System user transfers the data files (the lists of coordinates and time stamps) from the hand-held device to a personal computer (PC). On the PC screen, each input data point is represented by a dot in relation to the PDA's touch screen area. Subsequent to the recording process, the user can identify the input data according to the corresponding areas of the interface and can define the data (i.e., assign to them the corresponding predefined observation codes) by drawing polygons with the PC mouse to encircle the input data dots. Labels can then be assigned to the symbols accordingly. Figure 3 presents a PC screen shot of the signature or data definition process related to the FIT-System interface shown in Figure 2.

This signature or data definition procedure results in a list of event definitions, which include, for example, a data point's label and the related time stamp. The user can then initiate data processing according to a number of different models:

Single object, single channel. This model is based on the assumption that all events are related to a single object, such as the
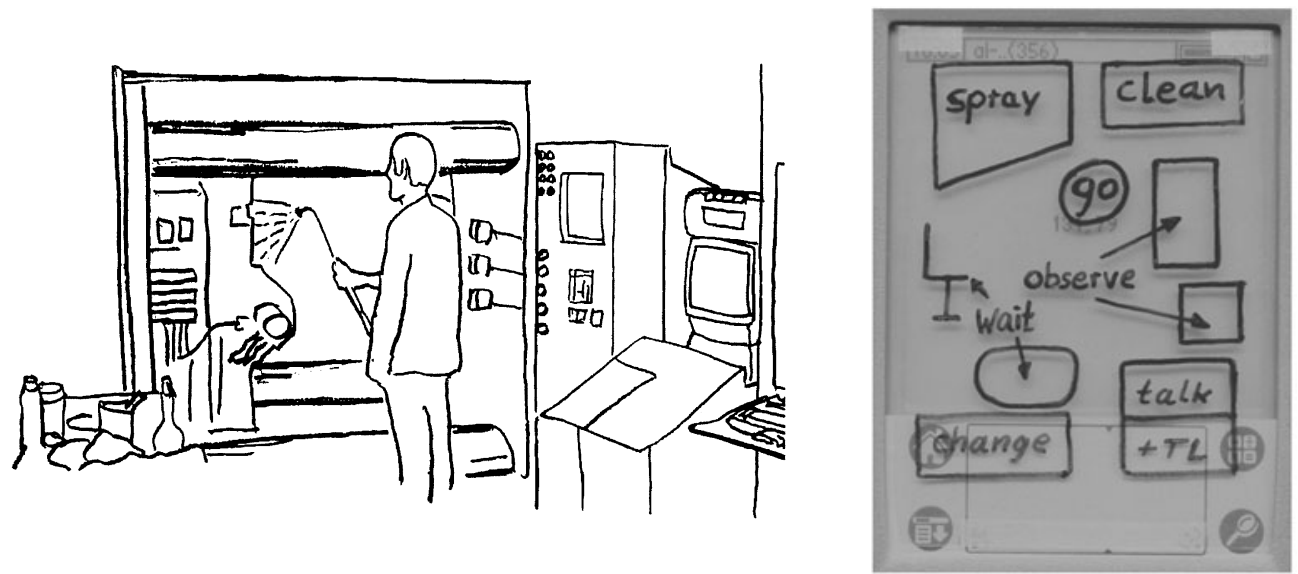

Figure 1. Workplace situation and FIT-System interface for online recording of sequential tasks in industrial production. The transparency overlay covers the liquid crystal display. 

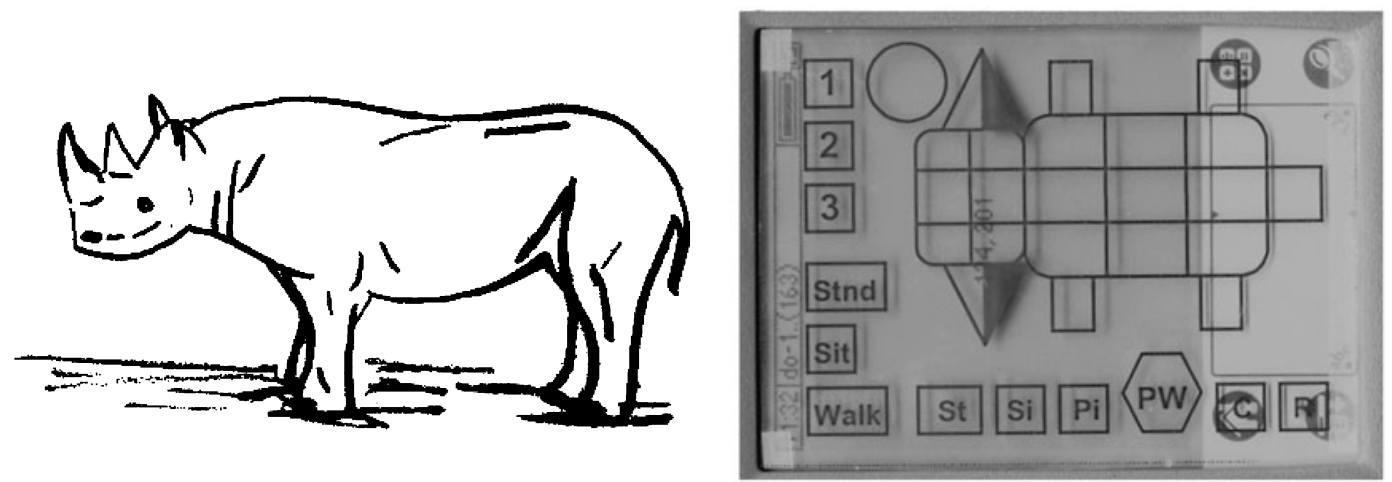

Figure 2. FIT-System interface for online recording of locations and events in the behavior of several oxpecker birds and a rhinoceros.

worker in the example shown in Figure 1. Events occur sequentially according to this model- that is, every event ends at the time the following one starts.

Several states, objects, and markers. A set of prefixes (such as the characters "*," "\#," and "!") in the definitions of the events tell the postprocessing data analysis software to distinguish between, for example, states (\#), objects (*), or markers (!) and activities or locations (see Figure 4).
Single object, concurrent events. An additional symbol, defined as a delimiter, allows the user to process data in "packages" of parallel or concurrent events. This model is described in more detail in the remainder of this article.

The table of defined event labels we used was written in ASCII format to facilitate its use with standard software; the FIT-System analysis software itself is an add-on for Microsoft's Excel spreadsheet program. Figure 4 shows an event-time list and its compila-

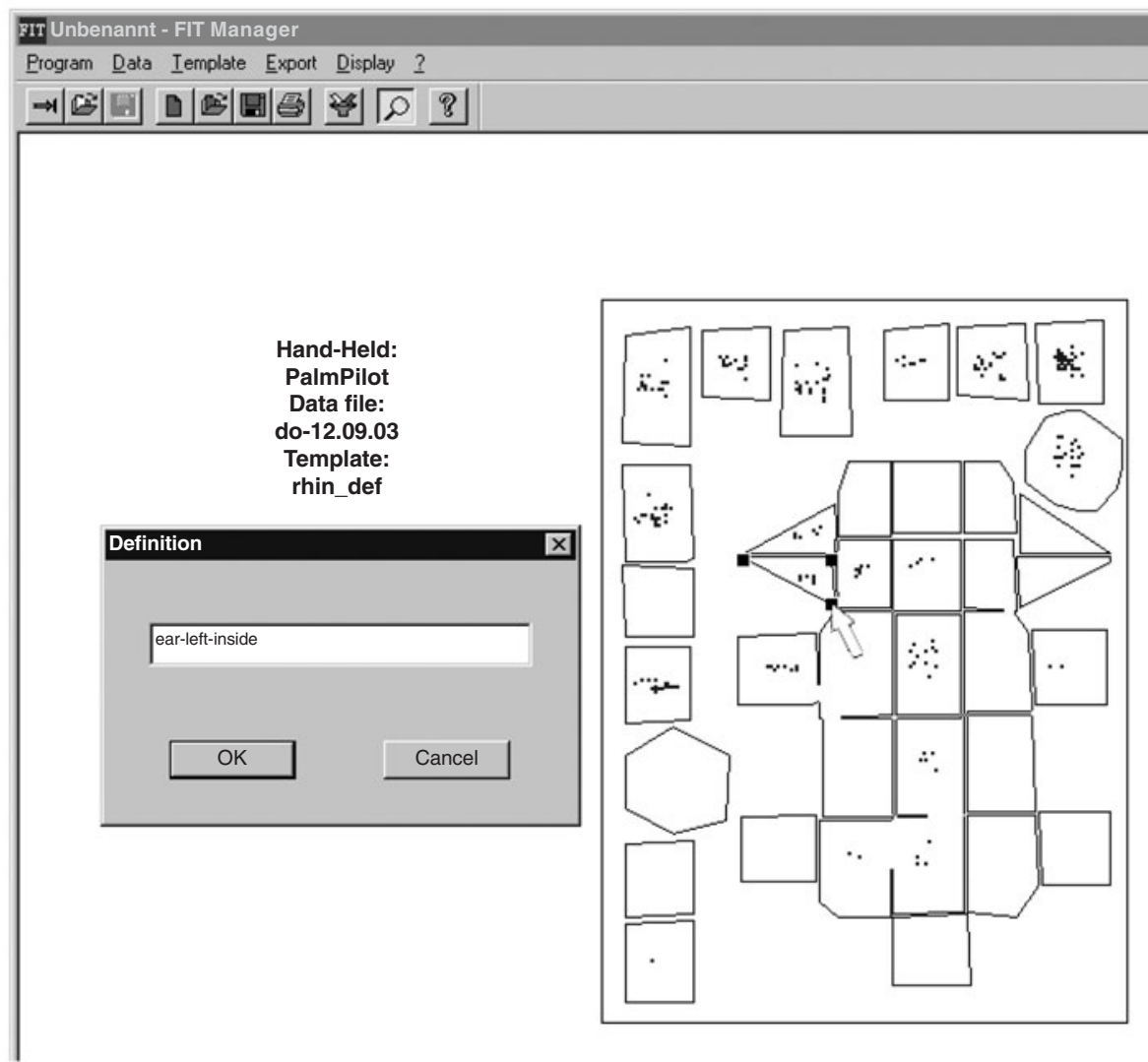

Figure 3. Signature of recorded events (locations, states, objects, activities, or markers) related to the application shown in Figure 2. 
A

\begin{tabular}{|c|l|c|c|}
\hline \multicolumn{1}{|c|}{ A } & B & C \\
\hline 1 & *rhino standing & $0: 18: 12$ & 2 \\
\hline 2 & \#bird 1 & $0: 18: 14$ & 2 \\
\hline 3 & feet-left-front & $0: 18: 16$ & 1 \\
\hline 4 & pick & $0: 18: 17$ & 2 \\
\hline 5 & \#bird 2 & $0: 18: 19$ & 1 \\
\hline 6 & feet-left-front & $0: 18: 20$ & 1 \\
\hline 7 & pick & $0: 18: 21$ & 4 \\
\hline 8 & \#bird 1 & $0: 18: 25$ & 1 \\
\hline 9 & ear-left-inside & $0: 18: 26$ & 1 \\
\hline 10 & pick & $0: 18: 26$ & 2 \\
\hline 11 & \#bird 2 & $0: 18: 28$ & 1 \\
\hline 12 & feet-left-front & $0: 18: 29$ & 1 \\
\hline 13 & pick & $0: 18: 30$ & 3 \\
\hline 14 & \#bird 2 & $0: 18: 33$ & 2 \\
\hline 15 & feet-left-front & $0: 18: 35$ & 1 \\
\hline 16 & pick & $0: 18: 36$ & 5 \\
\hline 17 & \#bird 1 & $0: 18: 40$ & 2 \\
\hline 18 & mid-mid & $0: 18: 42$ & 1 \\
\hline 19 & stand & $0: 18: 43$ & 3 \\
\hline 20 & \#bird 2 & $0: 18: 46$ & 1 \\
\hline 21 & !away & $0: 18: 47$ & 2 \\
\hline 22 & *rhino sit & $0: 18: 49$ & 1 \\
\hline 23 & \#bird 1 & $0: 18: 50$ & 1 \\
\hline 24 & feet-left-front & $0: 18: 51$ & 1 \\
\hline 25 & pick & $0: 18: 52$ & 0 \\
\hline
\end{tabular}

B

\begin{tabular}{|l|l|r|r|}
\hline \multicolumn{1}{|c|}{ A } & B & \multicolumn{1}{|c|}{ C } \\
\hline $\mathbf{1}$ & rhino standing & $0: 18: 12$ & 37 \\
\hline 2 & bird 1 - feet-left-front - pick & $0: 18: 14$ & 11 \\
\hline 3 & bird 2 - feet-left-front - pick & $0: 18: 19$ & 9 \\
\hline 4 & bird 1 - ear-left-inside - pick & $0: 18: 25$ & 15 \\
\hline 5 & bird 2 - feet-left-front - pick & $0: 18: 28$ & 18 \\
\hline 6 & bird 1 - mid-mid - stand & $0: 18: 40$ & 10 \\
\hline 7 & bird 2 - away & $0: 18: 46$ & 0 \\
\hline 8 & rhino sit & $0: 18: 49$ & 1 \\
\hline 9 & bird 1 - feet-left-front - pick & $0: 18: 50$ & 0 \\
\hline 1 1n & & & \\
\hline
\end{tabular}

C

$\begin{aligned} \# & \text { States } \\ * & \text { Objects } \\ ! & \text { Markers } \\ \text { none } & \text { Locations, Activities }\end{aligned}$

Figure 4. (A) Event-time list of raw data. (B) Its compilation. (C) Meaning of the prefixes. In Panels $A$ and B, column A shows the signature or event definition label, column B shows the time stamp (hh:mm:ss), and column C shows time differences in seconds.

tion based on a state-object-location-activity syntax that corresponds to the example in Figures 2 and 3.

Aggregation and visualization of the recorded data can be accomplished with the FIT-System software using five elements: (1) a table of basic descriptive statistics (frequencies, mean values, standard deviations, and quartiles); (2) an adjacency matrix of the graph; (3) a time line chart; (4) a box-plot time line diagram; and (5) a link chart-that is, a representation of event flow. The FITSystem also allows transferring data into the SPSS statistical software package.

\section{Syntax and Interface for the Recording of Concurrent Events in Anesthesia Work Processes}

To record concurrent events, we used a syntax that allows the user to enter all co-occurring events sequentially. To enable the software to distinguish between different lists of events, we added a symbol to the FIT-System's interface called "all"; the corresponding syntax can thus be called an "actions + all" syntax. The "all" symbol acts as a delimiter that marks the end of each event list. After entering all co-occurring events sequentially, the "all" symbol is activated by the user to indicate that a previously entered list of events all started at the same time. We should note that this delimiter has to be entered after every list of events, even if this list consists of only one element (i.e., there is no co-occurrence of events).

For example, in the case of simultaneously occurring actions A, $\mathrm{X}$, and $\mathrm{Z}$, the observer would tap the $\mathrm{A}, \mathrm{X}$, and $\mathrm{Z}$ symbols on the FIT-System's interface overlay and then tap the "all" symbol to indicate that $\mathrm{A}, \mathrm{X}$, and $\mathrm{Z}$ all started at the same time (i.e., the time that the first symbol was activated). As soon as the observer enters the first time stamp for a new event or list of events, the system marks the end of the previously occurring concurrent events. In our example, if only X continues after a few seconds of actions $\mathrm{A}, \mathrm{X}$, and
$\mathrm{Z}$ co-occurring, the user would tap the corresponding $\mathrm{X}$ symbol and then the "all" symbol to indicate that only one action continues at this point in time.

In Figure 5, the design of the FIT-System interface for our anesthesia example is depicted along with a mind map structure of the observation categories. The interface contains 41 symbols for the various observation categories, the "all" symbol, and an additional button to mark data entry errors.

We used the FIT-System's features described above to label the recorded data after the recording process, to distinguish between all recorded lists of concurrent events, and to assign the time stamp of the first element of such a list to each event in that particular list. Figure 6 presents an example of the output in the form of an eventtime list (including listings for the "all" symbol) and also shows a box-plot time line diagram illustrating the flow of events.

\section{Obtaining Data}

A trained observer who was familiar with the operating room environment and its procedures recorded anesthesiologists' action sequences throughout 23 cases involving a broad spectrum of surgical and anesthesia procedures. We did not systematically control for a possible reactivity to the presence of an observer. However, the observer had been in the field for several weeks prior to the actual data collection, and most participating anesthesiologists reported that they had gotten used to being observed during this time. Before starting each case, the anesthesiologist was explicitly instructed that patient care was paramount. At the end of each case, the observed anesthesiologist rated the case for whether or not it was routine.

\section{Level of Analysis}

This study is based on the theoretical framework of activity theory (Cole, 1996; Engeström, 1987), which distinguishes between three levels of human practice. Activity is driven by a complex mo- 
A B
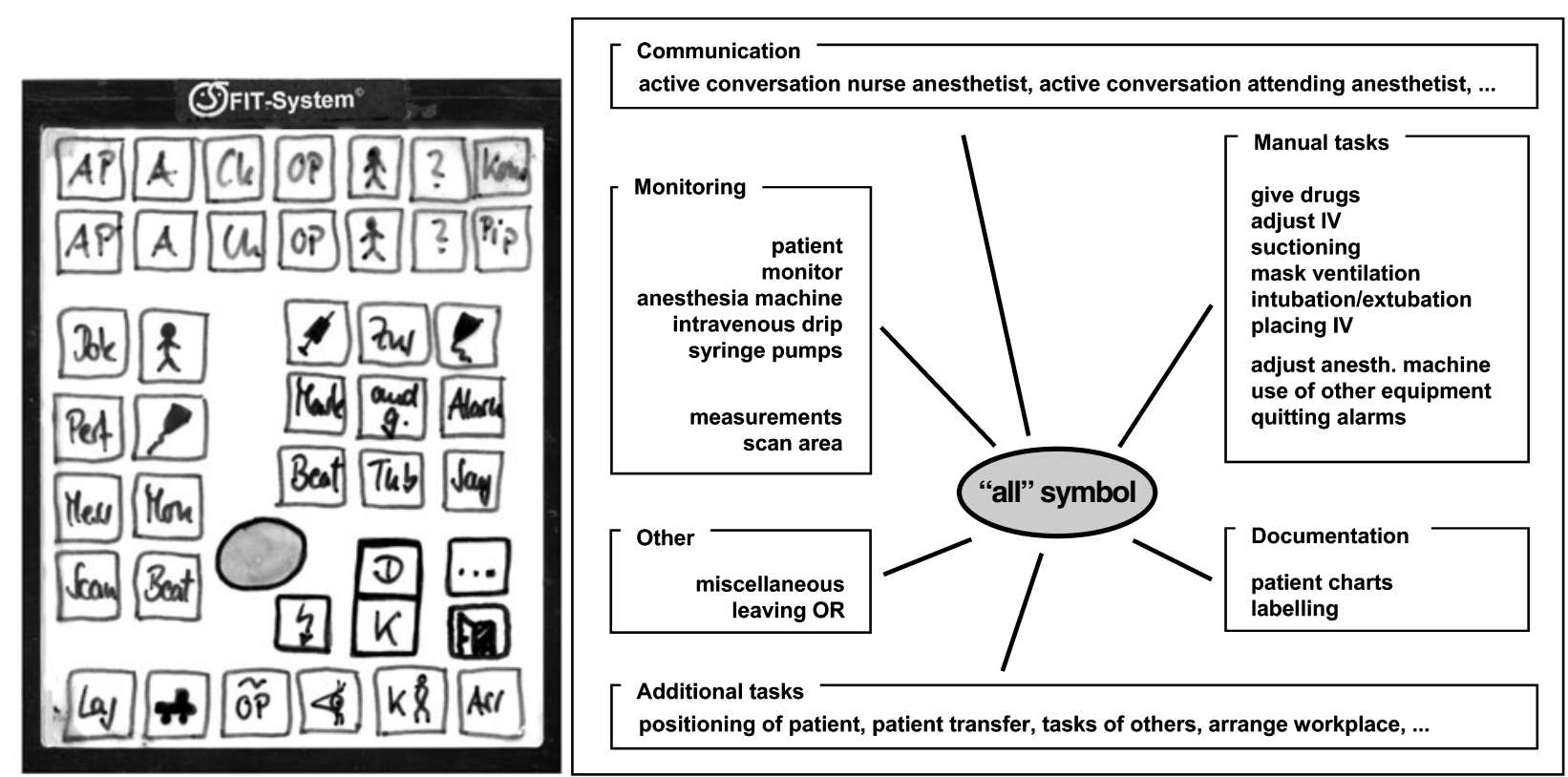

Figure 5. (A) FIT-System interface template for online recording of anesthesia-related concurrent events. (B) Its mind map structure.

tive that is collective in nature and that the individual actor is seldom aware of. An activity manifests itself in the form of goal-oriented individual actions. Actions in turn rely on incorporated operations that are influenced by the conditions under which they are performed. These concepts of activity theory give rise to certain methodological implications, such as a definition of the unit of analysis (Manser \& Wehner, 2002). According to this theoretical framework, only operations are directly observable. Moreover, because operations are influenced by the conditions under which they are performed, variations in the concrete conditions, such as the incidence of unexpected events, should correspond to variations at the level of operations. Therefore, observation is located on the level of operations that constitute action sequences of individual anesthesiologists. Our analysis focuses on the operational composition and potential co-occurrences of operations leading to the identification of different patterns. The complexity of action sequences may vary during the administration of anesthesia in response to, for example, the phase of the anesthesia process or the occurrence of unexpected events.

\section{Observation Categories}

On the basis of a literature review, several weeks of temporary attendance in the field, and discussions with anesthesia experts, Manser (2002) developed a set of 41 observation codes specific to the administration of anesthesia, each code representing a single operation. The five main categories structuring this set of observation codes were monitoring, manual tasks, communication, documentation, and additional tasks not belonging to the primary task of providing anesthesia (see Table 1).

\section{Interobserver Agreement}

Especially during the development of a new approach to behavioral analysis, it is important to assess interobserver agreement. In general, Cohen's kappa (Cohen, 1960) is used to assess interobserver agreement. In a context of concurrent events, though, we must remember that single observation codes are not mutually exclusive and may co-occur in various combinations. One way to compute Cohen's kappa would be to regard each combination of two or more observation codes as a "new code" and to assess observer agreement on each of these new codes. These results, however, would not be helpful in the definition of observation codes or in the training of observers. Manser (2002) therefore decided to assess interobserver agreement by computing Cohen's kappa for each observation code separately. Such a methodology means that a comparison of two independent observers coding during the same observation unit (in our case $1 \mathrm{sec}$ ) may result in agreement for one code and nonagreement for another if, for example, one observer codes an event as A and B, whereas another codes the same event as A and C. Moreover, if the observation task involves not only coding but also unitizing, a lag of a few seconds may also occur between the two observers (e.g., Observer 1 codes A, A, B, B, B, B, and Observer 2 codes A, A, A, B, B, B). In the study used as an example here, Manser (2002) applied a tolerance of $2 \mathrm{sec}$ in the analysis of interobserver agreement described by Bakeman and Gottman (1997). Finally, a weighted average was used to compute an overall score in order not to overestimate interobserver agreement in favor of rare events.

After the assessment of interobserver agreement described above (for details, see Manser, 2002), the results indicated a kappa of .68, which can be regarded as good agreement.

\section{Data Analysis}

To analyze the recorded data, we generated quantitative measures of frequency, duration, and percentage of total time for each observation code, as well as for empirical instances of co-occurrence. As we have indicated, this data analysis was carried out using FIT-System software based on Microsoft Excel spreadsheets. In order to describe the extent to which total activity, particular operations, and the number of concurrent operations fluctuated in the course of a specific action sequence, we calculated measures of action density 
Table 1

Observation Codes for Anesthesia Work Processes

\begin{tabular}{|c|c|}
\hline Main Categories & Detailed Observation Codes \\
\hline $\begin{array}{l}\text { Communication: } \\
\text { Active }\end{array}$ & $\begin{array}{l}\text { Active conversation with nurse anesthetist } \\
\text { Active conversation with attending anesthetist } \\
\text { Active conversation with surgeon } \\
\text { Active conversation with OR nurse } \\
\text { Active conversation with patient } \\
\text { Active conversation with others } \\
\text { Comment }\end{array}$ \\
\hline $\begin{array}{l}\text { Communication: } \\
\text { Responsive }\end{array}$ & $\begin{array}{l}\text { Responsive conversation with nurse anesthetist } \\
\text { Responsive conversation with attending anesthetist } \\
\text { Responsive conversation with surgeon } \\
\text { Responsive conversation with OR nurse } \\
\text { Responsive conversation with patient } \\
\text { Responsive conversation with others } \\
\text { Response to pager }\end{array}$ \\
\hline Monitoring & $\begin{array}{l}\text { Read patient record } \\
\text { Observe patient } \\
\text { Observe patient monitors } \\
\text { Observe anesthesia machine } \\
\text { Observe IV drip } \\
\text { Observe syringe pumps } \\
\text { Measurement } \\
\text { Scan area }\end{array}$ \\
\hline Manual tasks & $\begin{array}{l}\text { Place IV } \\
\text { Give drugs } \\
\text { Adjust IV } \\
\text { Mask ventilation } \\
\text { Intubation/extubation } \\
\text { Adjust anesthesia machine } \\
\text { Use of other equipment } \\
\text { Quitting alarms } \\
\text { Suctioning }\end{array}$ \\
\hline Documentation & $\begin{array}{l}\text { Patient chart } \\
\text { Labeling (drugs, drips) }\end{array}$ \\
\hline Additional tasks & $\begin{array}{l}\text { Positioning of patient } \\
\text { Patient transfer } \\
\text { Tasks of others (circulating nurse, orderly) } \\
\text { Arrange workplace } \\
\text { Conversation with observer } \\
\text { "Just looking" }\end{array}$ \\
\hline Other & $\begin{array}{l}\text { Miscellaneous } \\
\text { Leaving OR (with possible audiovisual contact with OR) }\end{array}$ \\
\hline
\end{tabular}

for the overall observation period as well as for different phases of an anesthesia case.

Action density indicates the number of concurrent events in a given time frame. The baseline for action density is by convention 100 , meaning that only one observation code occurred during a period with an action density of $100 .{ }^{1}$ Any empirical co-occurrence of behavioral events would cause an increase in action density above the defined baseline. If, for example, two codes co-occurred for the whole of a given time period, that action density would equal 200. If the two codes co-occurred for only two thirds of that time period, the resulting action density would be 167 .

\section{RESULTS}

In the following discussion, we will focus on the occurrence of concurrent events or overlapping operations in the course of administering anesthesia, using two different cases as examples. We will also highlight variations in action density corresponding to different phases of anesthesia and the occurrence of unexpected events.
In Figure 7, the structure of overlapping operations is visualized by using as an example about 2 min of an action sequence observed during anesthesia induction.

It is obvious that overlapping operations are quite frequent during this episode. If we do not consider the duration of an event (i.e., "Manual tasks" and "Monitoring" from 9:22 to 9:34 would be one event, and so would "Manual tasks," "Monitoring," and "Communication" from 9:44 to 9:49), 43\% of all observed events included concurrent tasks-that is, two or more overlapping operations. However, events involving such co-occurring observation codes (and especially more than two such codes) had generally shorter durations than those involving a single observation code.

Action density helps to reveal the extent to which total activity and the number of operations fluctuate during the action sequences observed. Figure 8 shows the distribution of action density and of the main behavioral categories (i.e., communication, monitoring, manual tasks, 


\begin{tabular}{|l|l|l|}
\hline Event (input in event recorder) & $\begin{array}{l}\text { Time } \\
\text { Stamp }\end{array}$ & $\begin{array}{l}\text { Time } \\
\text { Difference }\end{array}$ \\
\hline Adjust anaesthesia machine & $1: 53: 12$ & 1 \\
\hline All & $1: 53: 13$ & 10 \\
\hline Arrange workplace & $1: 53: 23$ & 0 \\
\hline All & $1: 53: 23$ & 18 \\
\hline Responsive conversation with surgeon & $1: 53: 41$ & 1 \\
\hline Arrange workplace & $1: 53: 42$ & 1 \\
\hline All & $1: 53: 43$ & 4 \\
\hline Observe patient monitors & $1: 53: 47$ & 0 \\
\hline Arrange workplace & $1: 53: 47$ & 1 \\
\hline All & $1: 53: 48$ & 5 \\
\hline Adjust anaesthesia machine & $1: 53: 53$ & 0 \\
\hline All & $1: 53: 53$ & 3 \\
\hline$\ldots$ & $\ldots$ & $\ldots$ \\
\hline
\end{tabular}

from: $1: 53: 12$

to: $1: 57: 26$

grid width [s]: 1

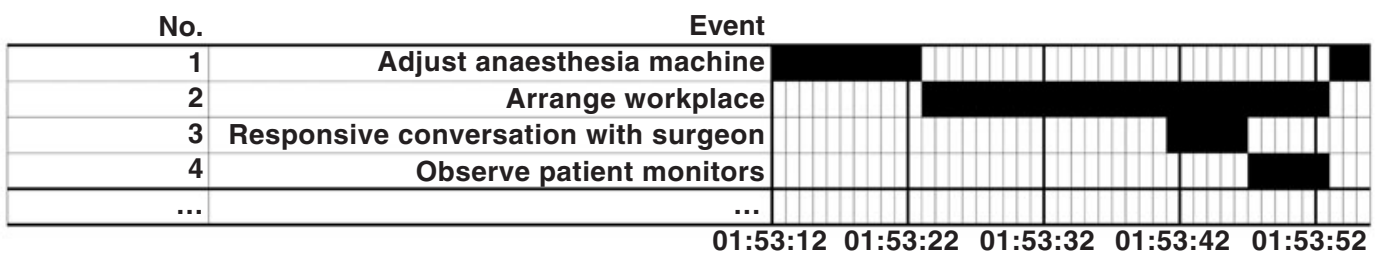

Figure 6. Event-time list, including labels describing the meaning of the syntax, and the box-plot time line diagram of recorded concurrent events.

and documentation) during an anesthesia case, which was rated as routine by the anesthesiologist. Because of frequent short-term fluctuations in the raw data, the moving average of action density of the previous $5 \mathrm{~min}$ is charted in this figure.

The results of our case analyses show that the action density during the application of anesthesia to a patient exhibits a characteristic and distinct distribution. For ex- ample, two periods that are characterized by increased action density are the induction of and the emergence from anesthesia (i.e., putting the patient to sleep and waking the patient up). The particular behavioral categories also display a distinct distribution in the course of the anesthesiologist's action sequence. For example, communication is most frequent during the induction of and the emergence from anesthesia, points at which sub-

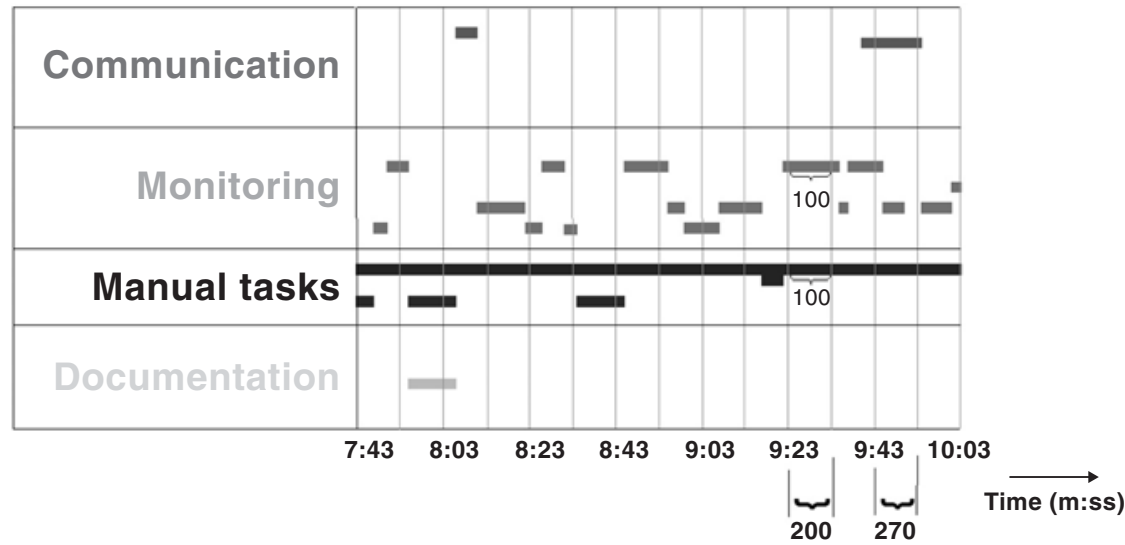

Figure 7. The structure of concurrent events during an episode of 2:20 min during induction of anesthesia in case 0153 . 

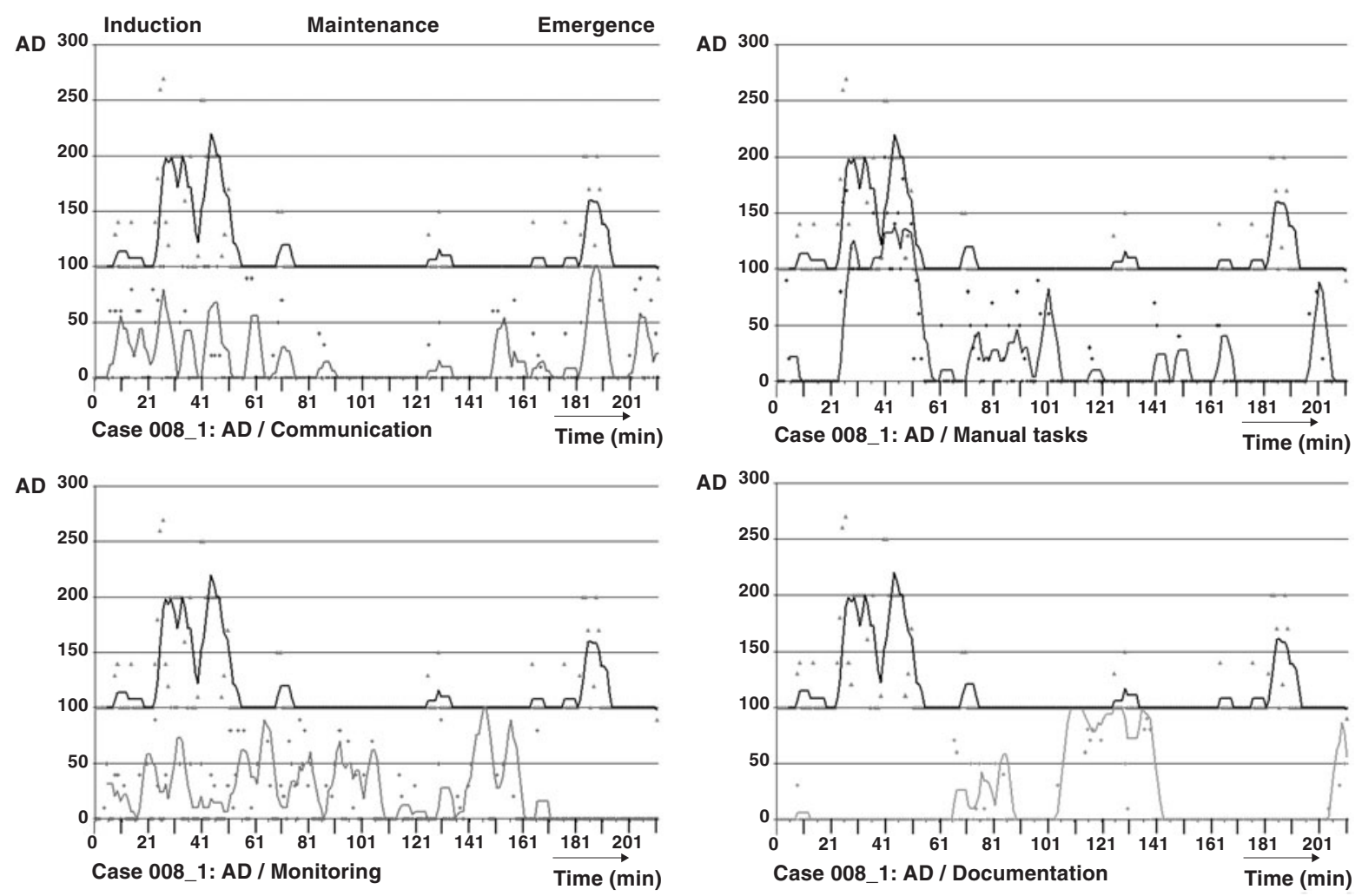

Figure 8. Variations in action density (AD)— that is, in the total number of overlapping operations — and in the relative numbers of communication, manual task, monitoring, and documentation operations during the course of case 008_1. (Graphs show the moving average over the previous 5 min.)

stantial coordination between team members takes place. Moreover, throughout most cases included in this study, there was an increase in the percentage of time spent monitoring operations while maintaining anesthesia during actual surgery, in comparison with during the induction of or the emergence from anesthesia, whereas the percentage of manual measures taken decreased during the maintenance phase. The highest percentage of manual tasks can be observed during the induction phase, whereas documentation becomes most concentrated during the maintenance phase. Only operations related to monitoring show a rhythmic curve throughout the entire anesthesia case. These results are supported by almost all cases involving general anesthesia that were included in this study. Moreover, overlapping operations have a prominent position when anesthetists deal with the various requirements relating to unexpected events (Manser $\&$ Wehner, 2002).

\section{DISCUSSION}

\section{Concurrent Events and Action Density}

The results presented here support the descriptive validity of action density as an indicator of the frequency of concurrent events, in our example overlapping operations in complex action sequences relating to adminis- tering anesthesia. First, the results show a considerable number of co-occurrences of observation codes that would have been neglected using most observation methods. Second, action density shows a characteristic and distinct distribution during the course of anesthesia.

As they have been described in the literature concerning the organization of multiple actions on an individual or a group level, concurrent actions may occur because of increased task complexity (Fuhrer, 1984; Kaminski, 1973; Tschan, 1990). Thus, we regard variations in action density as an indicator helpful for describing how health care professionals adopt to the varying complexity of task requirements in the course of anesthesia administration, especially in the case of unexpected complications. Moreover, the variations in action density revealed in our data fit very well with the results of research on workload (Gaba \& Lee, 1990) during the administration of anesthesia.

\section{Event Recording}

One of the advantages of using a PDA for event recording is that one can input data simply by tapping the pressure-sensitive screen rather than by using a separate keyboard. In general, the ease of use of the touch screen's interface depends on the ergonomic design of the GUI and on user involvement in the design process (International Organization for Standardization, 1999). In com- 
parison with current PDA-based event recording systems, the FIT-System is unique in the way it provides users with the autonomy to design their own GUIs (Table 2).

The GUI design method of the FIT-System allows the user to make the GUI a map of the situation to be observed instead of simply a symmetrical matrix of buttons. It should be mentioned that users of the FIT-System require no interaction with a PDA or PC to design their GUI template. Other PDA-based event recording systems force users to configure their coding scheme and label their buttons on the PC before downloading the template onto the PDA (e.g., Pocket Observer) or to perform all interactions for configuration in dialog with the PDA software. In comparison with these other PDA systems, the paper-and-pencil technique used with the FITSystem has the following advantages:

1. It is a fast, simple, and robust technique that supports a user's creativity and allows for the user's participation in ergonomic design (Noro \& Imada, 1991).

2. It enables the user to design the GUI without restrictions in the use of text, graphics, or color. No specific requirements of brightness, contrast, or colors are needed when creating the PDA display.

3. It facilitates the prototyping process of the coding scheme because it allows changes to be made online, without PDA interaction, and different GUI templates of the iterative design process to be compared side by side.

4. When used with some PDAs (i.e., those running the Palm OS), the GUI template has the advantage of providing $20 \%$ more interface area, because the template covers the "graffiti" field and allows it to be used as an input area (see Figures 1 and 2).

5. Any kind of symbol or icon can be created on the interface template and any kind of boundaries for the pointing area, whereas other systems are more restricted. For example, the ActoPalm system allows the design of circular buttons, but only the inner rectangular area of the circular form is active for pen-stroke recognition.

6. There is room for at least 50 icons (each $7 \times 7 \mathrm{~mm}$ ) on the template area, which covers $5.5 \times 7.0 \mathrm{~cm}$ (PalmOne OS device) or $6.5 \times 8.0 \mathrm{~cm}$ (Apple Newton device).

The fully adaptable layout of the GUI and the free arrangement of icons support a customizable relationship between the mapping of the symbols on the interface and the individual observation situation. For example, in Figure 1 the workplace layout is designed relating to tasks the worker has to perform at several locations. On the FIT-System interface, the corresponding symbols are arranged in a way that matches this work setting. To give another example, in Figure 2 the different areas of the rhinoceros's body an oxpecker bird might sit on are depicted on the GUI using a schematic drawing of the rhinoceros. We believe that this kind of customization of an interface reduces the observer's mental workload by reducing the effort required to memorize the syntax and symbols.

We experienced the problem that a user might not remember whether a certain event had already been entered or not. The FIT-System resolves this issue by allowing multiple inputs of the same event (see, e.g., Figure 4A, rows 11 to 16). The data compilation process is programmed to subsume and compress redundant input data. We assume that this interaction is faster than scanning the PDA screen for visual feedback such as, for example, whether the area around or under a specific symbol or button is highlighted.

Both PDA-based online recording and video analysis systems have several advantages and disadvantages, depending on the observation task at hand. One economic advantage of online recording is the 1:1 ratio of coding time to observed behavior, because the coding (identification and recording of the events) takes place in the same time frame as the observed behavior. One may argue, however, that there is no need for the observer to be present during video capture and that coding from videotape can also be done in a 1:1 ratio. For example, one can use the FIT-System or another event recording system in front of a video screen instead of being present in the real situation. This technique has the advantage of providing a reproducible data source, which is especially beneficial when assessing interobserver agreement. We evaluated this argument and made these findings.

1. Identifying events is often difficult when analyzing videotapes because of the fixed camera perspective and the reduction of the environment to a two-dimensional picture. During online recording, in contrast, the observer may continuously change his or her perspective by (minor) eye, head, or body movements.

2. In analogy with the differences between face-toface meetings and video conferencing, we assume that the online observer uses a broader spectrum of modalities than the two (visual and auditory) captured on videotape. Moreover, we found that during online recording there is more contextual information available than on video and that this additional information is needed for a correct coding of events in complex behavioral processes.

Furthermore, in many situations, such as operating rooms or emergency wards, it is difficult or even impossible to get approval for video recording.

Table 2

GUI Design of Some Current PDA-Based Recording Systems

\begin{tabular}{ll}
\hline \multicolumn{1}{c}{ GUI Design Process Involves } & \multicolumn{1}{c}{ System } \\
\hline Changing predefined labels and fixing arrangement of buttons & Pocket Observer (Noldus, Inc., www.noldus.com) or \\
& EthoScribe (Tima Scientific, www.tima.sci.com) \\
Creating, labeling, and arranging two types of buttons (boxes and circles) & ActoPalm (Kerguelen, 1999) \\
Free creation and arrangement of any types of icons and other GUI elements & FIT-System (Held, 1999) \\
\hline
\end{tabular}




\section{CONCLUSION}

In conclusion, we found evidence that concurrent events play an important role in complex behavioral processes. In our experience, the FIT-System is a practical and feasible means of overcoming the methodological problems that have previously been experienced when recording concurrent events in complex settings.

In the field of anesthesia, our study was the first of its kind. By analyzing complex action sequences in detail and focusing on concurrent events, it established a sound basis for improvements in task design and training. Nevertheless, further investigation into the operational composition of action sequences is needed. Additional research should be done, for example, on the effects of qualitatively different combinations of overlapping operations in action sequences on human performance in complex work environments.

None of the information presented here should be interpreted as an argument for restricting the analysis of complex behavioral processes to medical work alone. Rather, we would emphasize the need to integrate numerous settings in order to provide a well-rounded and balanced system for investigating human activity and the role of concurrent events in complex behavioral processes.

\section{REFERENCES}

BaKeman, R., \& GotTMAn, J. M. (1997). Observing interaction: An introduction to sequential analysis (2nd ed.). Cambridge: Cambridge University Press.

Cohen, J. (1960). Coefficient of agreement for nominal scales. Educational \& Psychological Measurement, 20, 37-46.

CoLe, M. (1996). Cultural psychology. Cambridge, MA: Harvard University Press.

ENGESTRÖM, Y. (1987). Learning by expanding: An activity-theoretical approach to developmental research. Helsinki: Orienta-Konsultit.

FuHRER, U. (1984). Mehrfachhandeln in dynamischen Umfeldern: Vorschläge zu einer systematischen Erweiterung psychologischhandlungstheoretischer Modelle. Göttingen: Hogrefe.

GABA, D. M., \& LEE, T. (1990). Measuring the workload of the anesthesiologist. Anesthesia \& Analgesia, 71, 354-361.

HeLD, J. (1999). Fitting the mental model: A new technique for computerised event recording. In D. Harris (Ed.), Engineering psychology and cognitive ergonomics (pp. 293-300). London: Ashgate.
HeLD, J. (2003). Method and device for manual recording of various events or states. U.S. Patent No. 6525712B1.

Held, J., Brüesch, M., Zollinger, A., Pasch, T., \& Krueger, H. (2002). Beteiligungsorientierte Arbeitsanalyse [Participation-oriented work analysis]. Anaesthesist, 51, 110-115.

InTERNATIONAL ORganizATION FOR STANDARDIZATION (1999). Humancentred design processes for interactive systems (ISO 13407). Geneva: Author.

KAMINSKI, G. (1973). Bewegungshandlungen als Bewältigung von Mehrfachaufgaben. Sportwissenschaft, 3, 233-250.

Kennedy, P. J., Feingold, A., Wiener, E. L., \& Hosek, R. S. (1976). Analysis of tasks and human factors in anesthesia for coronary-artery bypass. Anesthesia \& Analgesia, 55, 374-377.

Kerguelen, A. (1999). ActoPalm 1.0 pour PalmPilot [Technical note]. Université de Toulouse-Le Mirail, Laboratoire Travail et Cognition.

MANSER, T. (2002). Dichte als Texturmerkmal komplexer Arbeitstätigkeiten: Zur Analyse von Überlagerungen im Handlungsverlauf am Beispiel der Narkoseführung. Zürich: Zentralstelle der Studentenschaft der Universität Zürich.

MANSER, T. (2003). Dichte als Merkmal komplexer Arbeitstätigkeiten. In T. Manser (Ed.), Komplexes Handeln in der Anästhesie (pp. 100174). Pabst: Lengrich.

Manser, T., \& Wehner, T. (2002). Analysing action sequences: Variations in action density in the administration of anesthesia. Cognition, Technology, \& Work, 4, 71-81.

Noro, K., \& Imada, A. (1991). Participatory ergonomics. London: Taylor \& Francis.

Tschan, F. (1990). Organisationen als sich zielgerichtet verhaltende Systeme: Entwicklung eines theoriegeleiteten Beschreibungssystems im Rahmen einer Fallstudie. Unpublished dissertation, Universität Bern.

Weinger, M. B., Herndon, O. W., \& Gaba, D. M. (1997). The effect of electronic record keeping and transesophageal echocardiography on task distribution, workload, and vigilance during cardiac anesthesia. Anesthesiology, 87, 144-155.

Weinger, M. B., Herndon, O. W., Zornow, M. H., Paulus, M. P., GABA, D. M., \& DALlEN, L. T. (1994). An objective methodology for task analysis and workload assessment in anesthesia providers. Anesthesiology, 80, 77-92.

\section{NOTE}

1. This baseline has been defined on the basis of the assumption that the behavioral codes used are exhaustive. If they are not, action density can drop and reach 0 when no codes occur in a given time period.

(Manuscript received September 24, 2002; revision accepted for publication May 13, 2004.) 